\title{
Surgical Correction of Craniosynostosis. A Single Institution`s Outcome Analysis of 70 Patients
}

\author{
Evangelos G. Kilipiris 1,*, Frantisek Horn², Michal Petrik3, Michal Kabat4, Jan Trnka ${ }^{5}$, Peter Stanko ${ }^{6}$ \\ 1 Oral and Maxillofacial Surgery Residency Program, PGY4, Comenius University, Faculty of Medicine, Bratislava, Slovak Republic \\ 2 Department of Pediatric Surgery, Division of Pediatric Neurosurgery, Comenius University, Faculty of Medicine, Pediatric University \\ Hospital and Polyclinic, Bratislava, Slovak Republic $(P h D)$
3 Department of Pediatric Surgery, Division of Pediatric Neurosurgery, Comenius University, Faculty of Medicine, Pediatric University \\ 3Department of Pediatric Surgery, Division of Pediatric
Hospital and Polyclinic, Bratislava, Slovak Republic \\ Hospital and Polyclinic, Bratislava, Slovak Republic
4Department of Pediatric Surgery, Division of Pediatric Neurosurgery, Comenius University, Faculty of Medicine, Pediatric University \\ Hospital and Polyclinic, Bratislava, Slovak Republic $(P h D)$ \\ ${ }_{5}$ Chair of the Department of Pediatric Surgery, Comenius University, Faculty of Medicine, Pediatric University Hospital and Polyclinic, \\ Bratislava, Slovak Republic (CSc, Assoc Prof) \\ ${ }_{6}^{6}$ Chair of the Department of Stomatology, Oral and Maxillofacial Surgery, Comenius University, Faculty of Medicine and St. Elizabeth \\ Oncologic Institute, Bratislava, Slovak Republic (PhD, Prof)
}

\section{ABOUT ARTICLE}

Article history:

Paper received 3 December 2017

Accepted 6 December 2017

Available online 28 December 2017

Keywords:

Cranial vault

Cranial sutures

Craniosynostosis

Synostosis

Scaphocephaly

Total cranial vault reconstruction

Trigonocephaly

Barrel-staving cuts

Fronto-orbital advancement

\begin{abstract}
A B S T R A C T
Purpose.

The goal of the current study is to provide outcome data for open cranial vault reconstruction at a single institution by a single craniofacial-neurosurgical team.

Patients and Methods.

A total of 70 patient records were reviewed. The inclusion criteria were patients less than 3 years of age undergoing primary surgery with open cranial vault reshaping and a minimum follow up time of 2 years.

Findings.

Of the 70 patients meeting the selection criteria ( 32 female, 38 male), 5 were syndromic and 65 nonsyndromic. Average age and weight were 8.8 months and $9 \mathrm{~kg}$ respectively. The oldest child was 21 months and the youngest 3.5 months at the time of surgery. The estimated blood volume lost was $35.8 \%$ of total calculated blood volume. Average surgical time was 223.2 minutes.

Conclusion.

Our review of 70 open repairs of patients with craniosynostosis demonstrates good long-term results with an overall low complication rate and represents open cranial vault reconstruction as a valuable method for repair of such defects.
\end{abstract}

\section{Introduction}

Craniosynostosis, the premature fusion of cranial sutures, occurs in approximately $3.5-4.5$ out of 10,000 live births worldwide [1]. It can affect one or multiple sutures, occur as an isolated defect or be associated with a craniofacial syndrome. Nonsyndromic craniosynostosis presents more commonly than syndromic craniosynostosis. Single suture synostosis results in head shape deformities with classic presentations depending on which suture is involved. Sagittal suture fusion results in scaphocephaly, the most common synostosis abnormality in Europe, following by metric suture fusion resulting in trigonocephaly. Unilateral

\footnotetext{
* This manuscript has not been presented

* Corresponding author. Department of Oral and Maxillofacial Surgery, Comenious University, Faculty of Medicine, Moskovska 15, Bratislava 81108, Slovak Republic Tel.: +421944715632, Fax: +421232249724 E-mail address: varonos@live.co.uk (E.G. Kilipiris) Instagram: @evangeloskilipiris
}

coronal suture fusion, more commonly, or lambdoid suture fusion, less commonly result in plagiocephalic head shapes, and bilateral coronal or lambdoid fusions present with brachiocephalic head shape deformities [2].

Intracranial hypertension, visual impairment, limitation of brain growth and neuropsychiatric disorders are associated with craniosynostosis. Generally, a greater functional disturbance appears in proportion to the number of sutures involved [3]. In surgically correctable cases, various approaches have been described. Historically, all repairs were performed via open transcranial approaches. Recently, interest in minimally invasive techniques, such as endoscopic suture release, spring assisted surgery, and distraction osteogenesis have been studied in an effort to potentially reduce surgical morbidity [4].

While inherent risks of open cranial vault reshaping exist, the past two decades have enjoyed advances in resorbable fixation, imaging modalities, and perioperative medical management. The purpose of this retrospective 
study is to provide a single institution's experience in types of craniosynostosis, management techniques, perioperative data and complication rates for open cranial vault reshaping at Kramare Pediatric University Hospital with Polyclinic in Bratislava.

\section{Patients and Methods}

The inclusion criteria for this study were patients less than three years of age undergoing primary surgery with open cranial vault reshaping and a minimum of 2 years follow-up. Based on inclusion criteria, 70 patients with craniosynostosis, treated with surgical correction between 2005 and 2015 by the institutional craniofacial and neurosurgical team were included. The surgical procedure of choice was single stage open transcranial vault reshaping with barrel-staving and orbital bandeau advancement as required for existing fronto-orbital dysmorphology. Resorbable plates with screws and absorbable sutures were used, based on their success and safety in pediatric craniofacial surgery [5].

All cases were performed by a single craniofacialpediatric neurosurgical team, and rotating anesthesiologists and pediatric intensivists assigned to the team. Patient medical records were used to assess the length of surgery, estimated blood loss, postoperative complications and average length of hospital stay.

A complete history and physical exam were performed along with computed tomography utilizing threedimensional reconstruction for pre-surgical planning. Standard monitoring using temperature probes, electrocardiography, capnography, and pulse oximetry
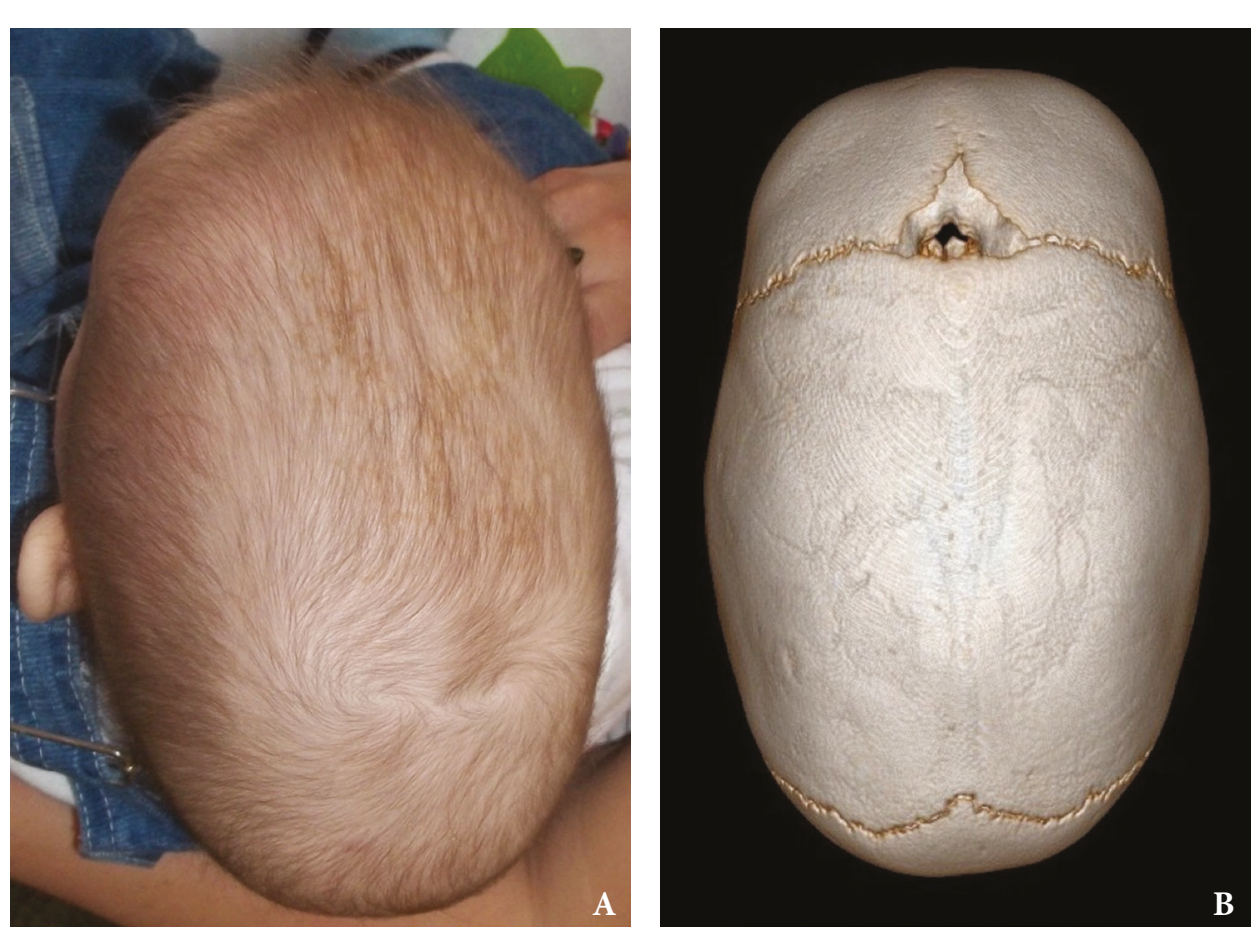

FIGURE 1. (A) Clinical picture of patient with scaphocephaly due to sagittal suture synostosis. (B) Preoperative 3D reconstruction CT scan demonstrating fusion of the sagittal suture. (Fig 1 continued on the next page.)

were employed. Induction was achieved with sevoflurane in most cases. The standard protocol included central venous access and an arterial line, hypotensive anesthesia, and packed red blood cell transfusions given at key portions of each case to correspond with anticipated blood loss.

Patients undergoing anterior cranial vault reshaping for metopic or coronal suture synostosis were placed supine in the pediatric horseshoe headrest. Those undergoing surgery for posterior or total cranial vault reshaping were placed prone with the neck slightly extended to allow access to the entire cranial vault. In prone cases, extra care in the way of foam padding was used to protect the globes.

All procedures employed a coronal incision with Raney clips for hemostatic assistance. Dissection was carried out in a subperiosteal plane to expose the necessary area for reshaping. Treatment of scaphocephaly consists of total cranial vault reshaping, with variations depending on the extent of fusion and which part of the sagittal suture is involved (Fig 1A-D). When the posterior half was fused, the patient was treated in the prone position with the posterior two thirds of the cranial vault reshaped. When the anterior half was fused, the patient was treated in the supine position with the anterior two thirds of the cranial vault reshaped, with or without superior orbital rim reshaping (Fig 2AC). Complete sagittal suture synostosis was treated at one operative setting in the prone position via total cranial vault reshaping. Typically sagittal synostosis procedures are performed between 6 and 9 months of age. In older children (older than 1 year) or children with a need for upper orbital reconstruction, the preference was to treat them in the supine position at one operative setting. (1) 

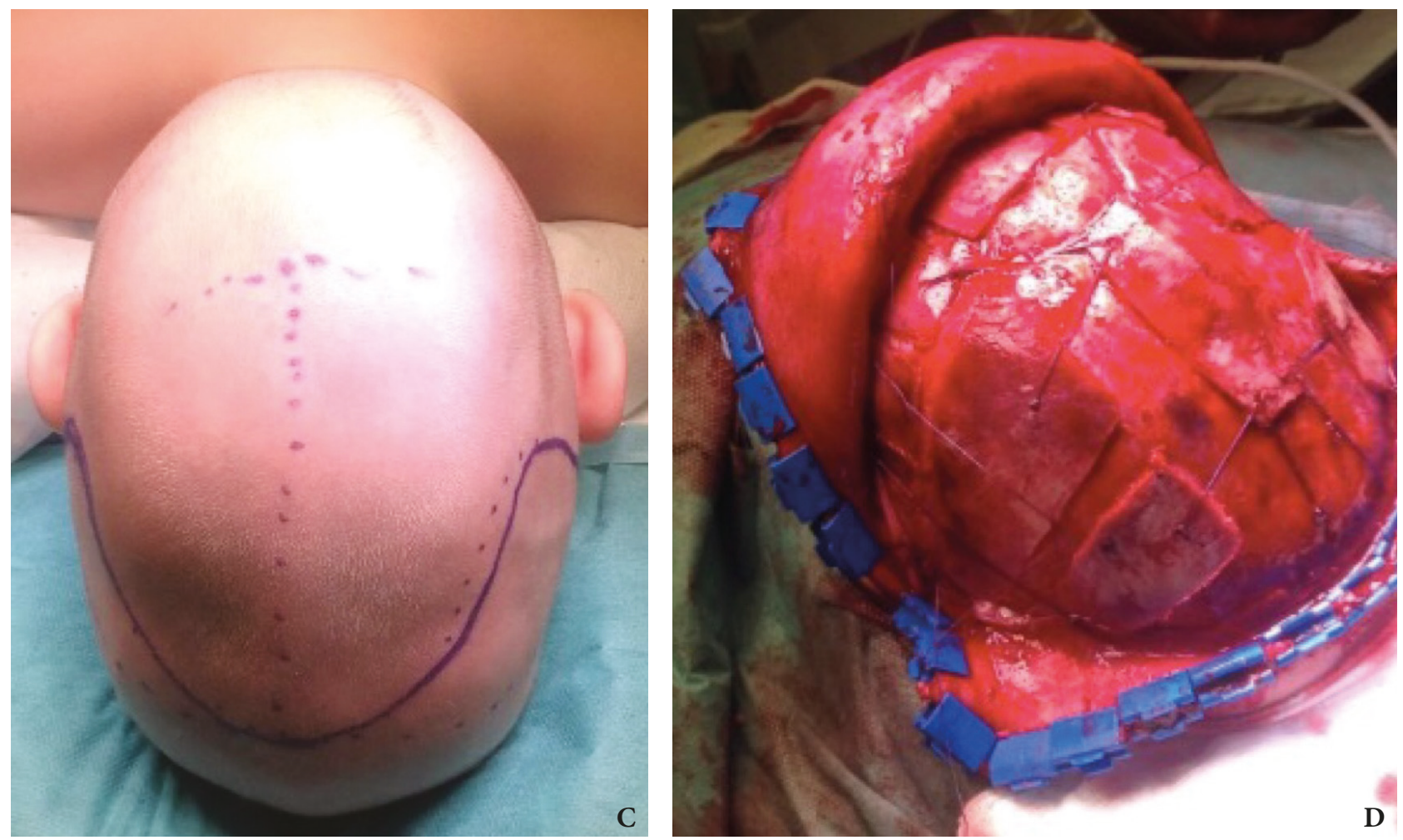

FIGURE 1. (cont'd). (C) Patient on prone position with proposed coronal incision marked. (D) Total cranial vault reconstruction with absorbable Vicryl sutures for fixation of bone flaps.
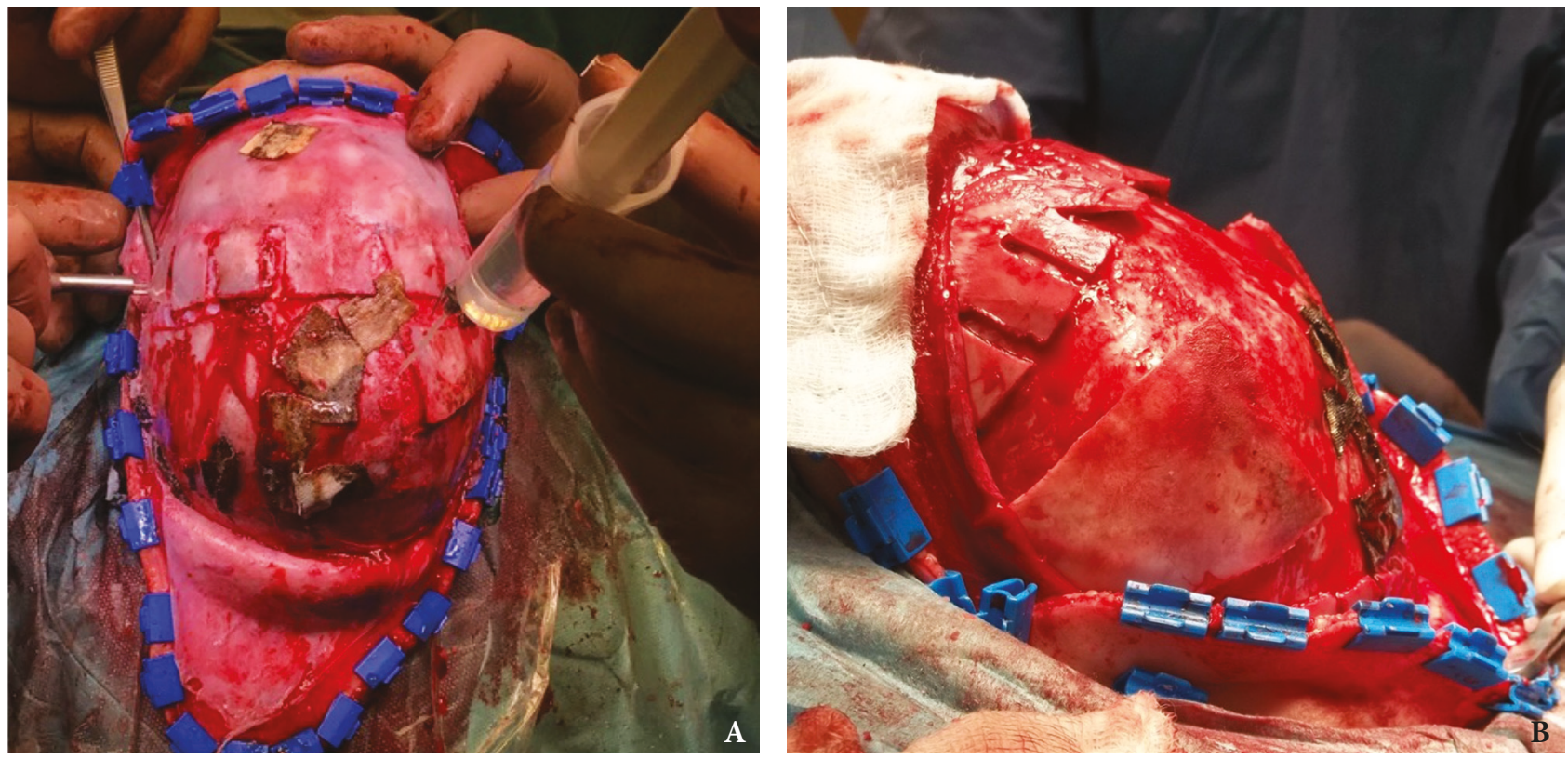

FIGURE 2. (A-C) Patient with sagittal suture synostosis and a normal supraorbital region on supine position after release of the fused suture and barrel-staving cuts to facilitate bitemporal widening. (Fig 2 continued on the next page.) 


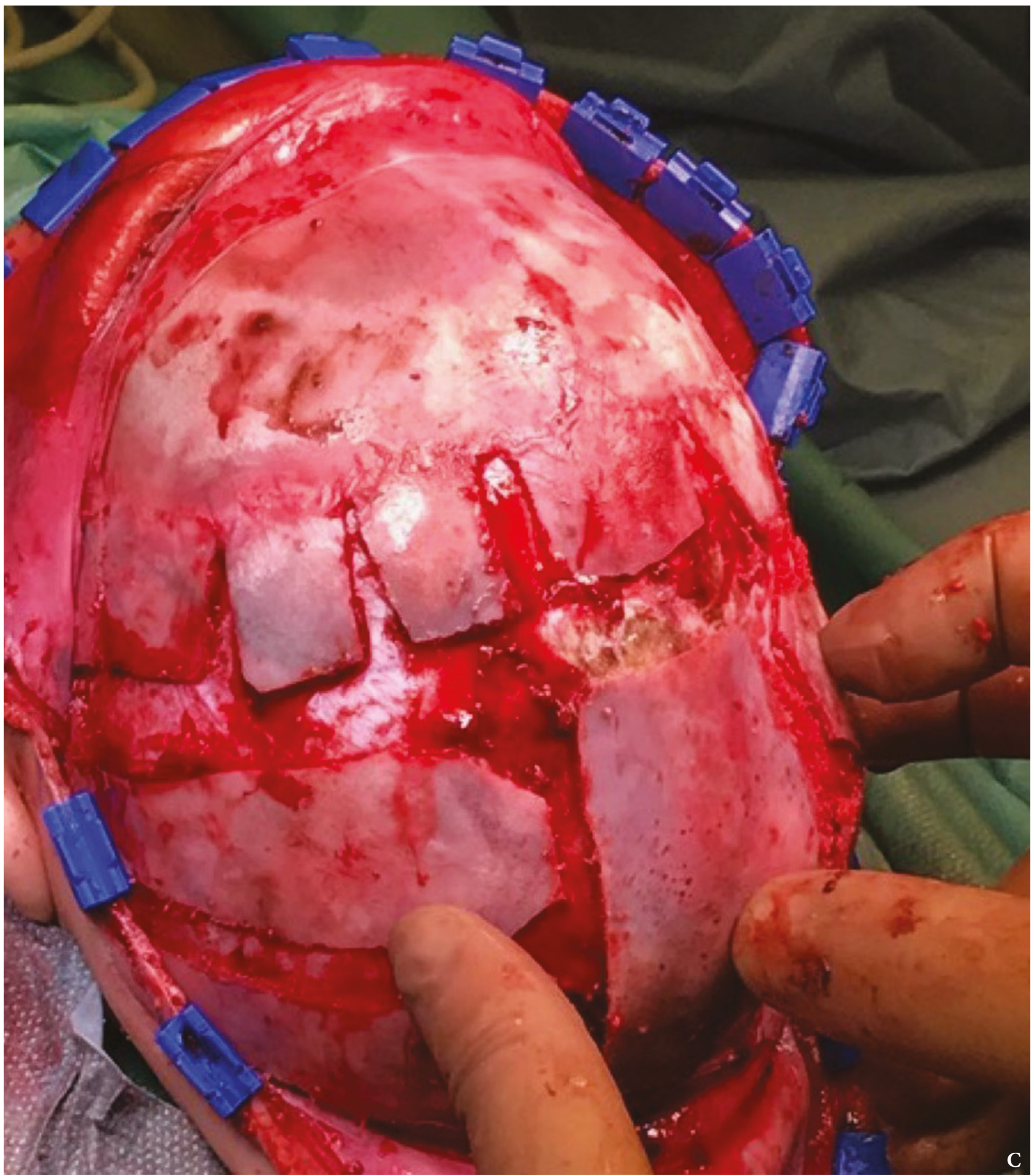

FIGURE 2. (cont'd). (A-C) Patient with sagittal suture synostosis and a normal supraorbital region on supine position after release of the fused suture and barrel-staving cuts to facilitate bitemporal widening.

Cases of bilateral coronal suture synostosis were treated with simultaneous frontal bone and bilateral orbital rim advancement. Correction of trigonocephaly involved metopic suture release, simultaneous bilateral rim advancements, and lateral widening via frontal bone advancement (Fig 3AG). Orbital hypotelorism was corrected by splitting the supraorbital unit in the midline and placing autogenous cranial bone grafts to increase the intraorbital distance. 

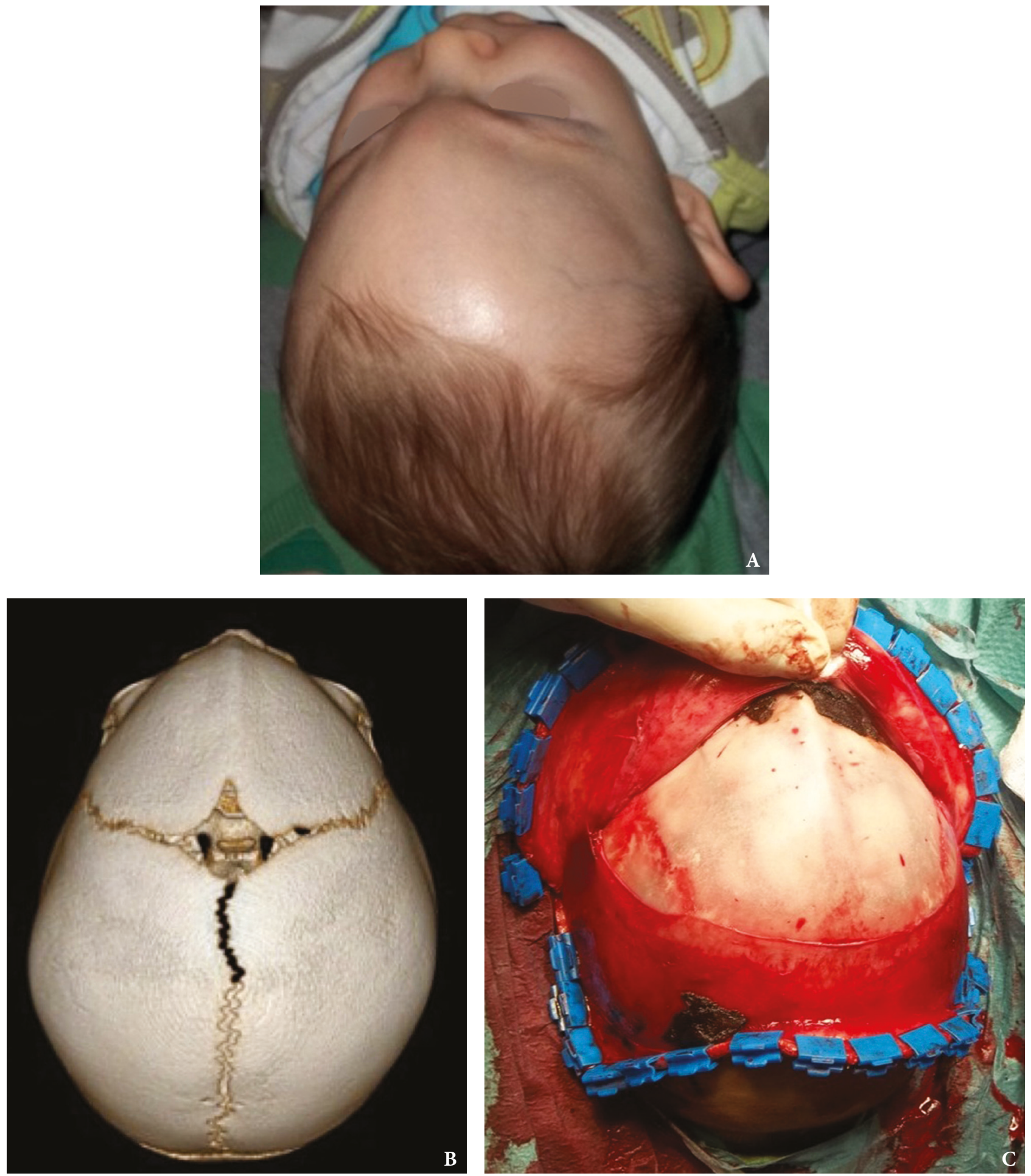

FIGURE 3. (A) A 9-month-old boy with metopic synostosis resulting in trigonocephaly. (B) 3D reconstruction CT scan showing fusion of the metopic suture. (C) Intraoperative view of the fused metopic suture. (Fig 3 continued on the next page.) 

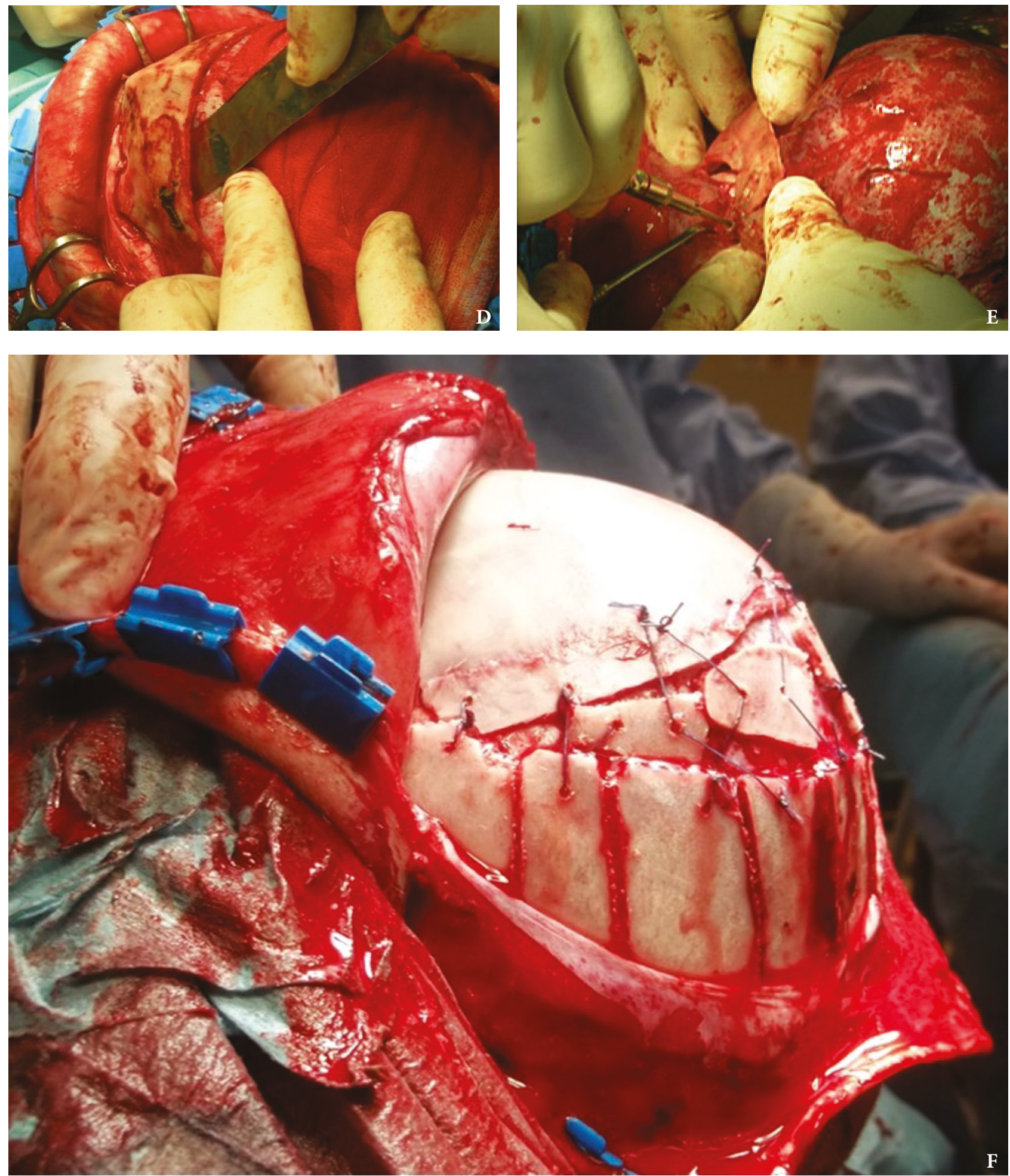

FIGURE 3. (cont'd). (D), (E) Bilateral superior orbital rim reshaping and advancement. (F) Anterior cranial vault reshaping. (Fig 3 continued on the next page.) 


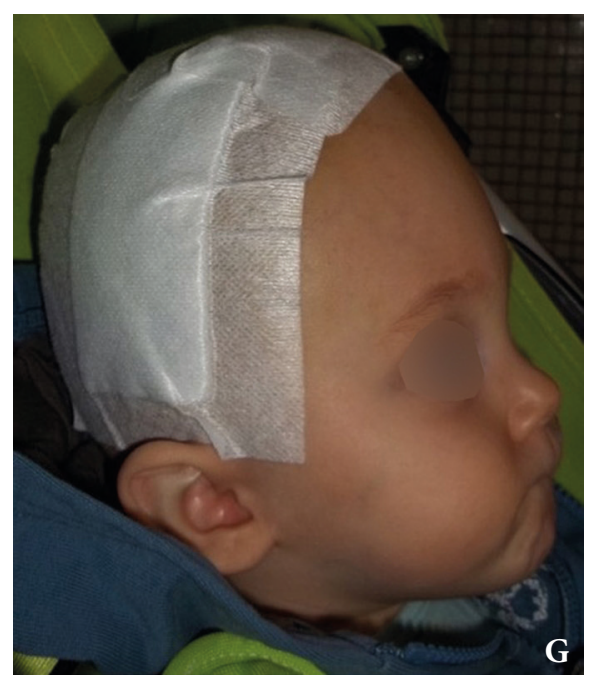

FIGURE 3. (cont'd). (G) Lateral view one week after reconstruction.

\section{Results}

Of the 70 patients meeting the selection criteria, the average age and weight at the time of surgery were 8.8 months and $9 \mathrm{~kg}$ respectively. The oldest child was 21 months and the youngest 3.5 months at the time of surgery. The most commonly synostosed suture was the sagittal followed by the metopic. The estimated blood volume (EBV) loss was on average $241.6 \mathrm{ml}$, and the surgical time $223.2 \mathrm{~min}$ (Chart 1). Average length of PICU stay was 3.17 days. Of the 70 cranial vaults 5 were associated with a syndrome and 65 were isolated synostosis (Table 1).

TABLE 1. Results - Articles Included in the Study and Relevance

\begin{tabular}{|l|l|}
\hline Perioperative Demographics & Number \\
\hline Average surgical age (months) & $8.9($ range $3.5-21)$ \\
\hline Average weight at time of surgery $(\mathrm{kg})$ & 9 (range 5.2-21) \\
\hline Estimated average blood loss $(\mathrm{ml})$ & $241.6($ range $90-2100)$ \\
\hline Average hospital stay (days) & 7.24 (range 5-16) \\
\hline Average surgical time (minutes) & 223.2 (range $72-442)$ \\
\hline
\end{tabular}

CHART 1. Estimated blood volume loss versus surgical time (minutes)

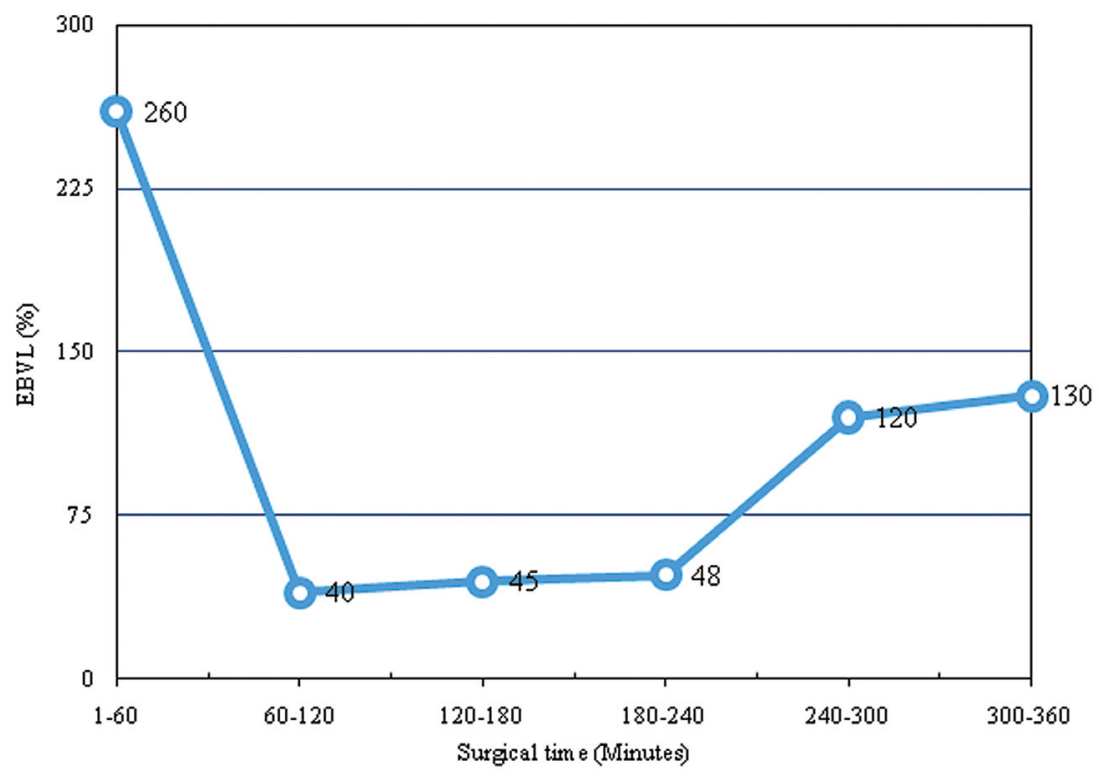


Complications included 1 subgaleal hematoma that was aspirated, 1 wound infection treated with oral cephalexin, 1 subgaleal abscess requiring drainage, 4 dural tears were repaired intraoperatively with Nurolon suture, and 1 case with sagittal sinus bleed (Table 2), (Fig 4).

TABLE 2. Number of complications.

\begin{tabular}{|l|l|}
\hline Summary of Complications & Number \\
\hline Subgaleal hematoma (Fig 4) & 1 \\
\hline Wound infection & 1 \\
\hline Subgaleal abscess & 1 \\
\hline Dural tear & 4 \\
\hline Sagittal sinus bleed & 1 \\
\hline
\end{tabular}

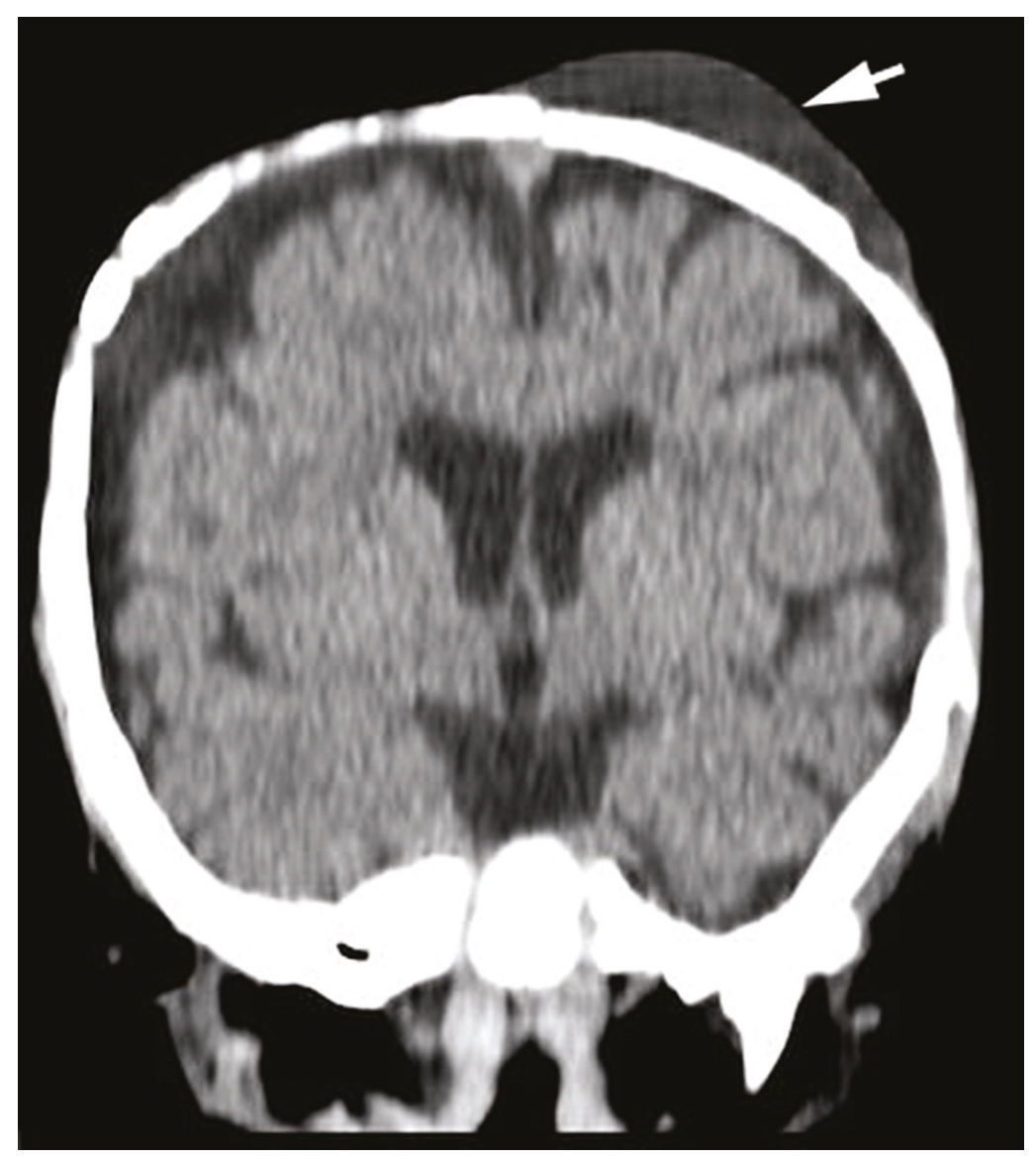

FIGURE 4. CT scan coronal projection of one patient developing a subgaleal hematoma (arrow) requiring drainage postoperatively.

Nearly half of the patients in the study (44\%) had a scaphocephalic head shape as a result of sagittal suture synostosis. Trigonocephaly was the second most common head shape deformity encountered in this study with $27 \%$ due to metopic suture synostosis. While the most common cause of plagiocephalic head shape is deformational, the most common form of synostotic plagiocephaly is a result of unilateral fusion of the coronal suture, with lambdoid synostosis occurring very rarely [6]. Whenever posterior plagiocephaly was encountered, positional or infant molding with or without congenital torticollis were ruled out. All cases of plagiocephaly in this series were the result of unilateral coronal synostosis. Out of 70 patients, 4 presented with a brachycephalic head shape due to fusion of the coronal sutures bilaterally. Of the 4 cases of brachycephaly in this study, the majority (3/5) of our syndromic patients fell in this category. Two patients had Crouzon syndrome and one patient Apert's syndrome.

Eight patients presented with fusion of more than one suture (Chart 2 and Table 3 ). Four patients demonstrated a fusion of the unilateral coronal suture in association 
with the sagittal suture. Three patients presented with fusion of the metopic and sagittal sutures, and one patient encountered fusion of the metopic suture together with the coronal sutures bilaterally. The procedure planned for treatment of the patients with unilateral coronal and sagittal suture fusion was orbital rim advancement along with bifrontal and unilateral parietal craniotomies for cranial vault reshaping. In the second group of patients, the metopic suture fusion resulted in frontal bossing and suture ridging but did not affect the orbital rims. For correction of the metopic suture fusion, direct frontal contouring was performed using a round bur. The sagittal suture was removed. Occipital and bilateral parietal craniotomies were performed. Barrel-staving cuts were developed to facilitate the final reshaping and bone flaps were fixated with absorbable Vicryl sutures. The only patient with fusion of metopic and bicoronal sutures was managed with anterior cranial vault reshaping, bilateral superior orbital rim advancements and bitemporal widening via barrel-staving osteotomies.

TABLE 3. Distribution of involved sutures and syndromic versus nonsyndromic patients.

\begin{tabular}{|l|l|}
\hline $\begin{array}{l}\text { 70 Total Patients } \\
(32 \text { Female, 38 Male) }\end{array}$ & Syndromic vs Nonsyndromic \\
\hline 34 Scaphocephalic & 65 Nonsyndromic \\
\hline 23 Trigonocephalic & 5 Syndromic ( 2 Apert, 3 Crouzon) \\
\hline 1 Plagiocephalic & \\
\hline 4 Brachycephalic & \\
\hline 8 Multiple Suture & \\
\hline
\end{tabular}

CHART 2. Distribution of head shape deformity and synostosis in total numbers.

87,5

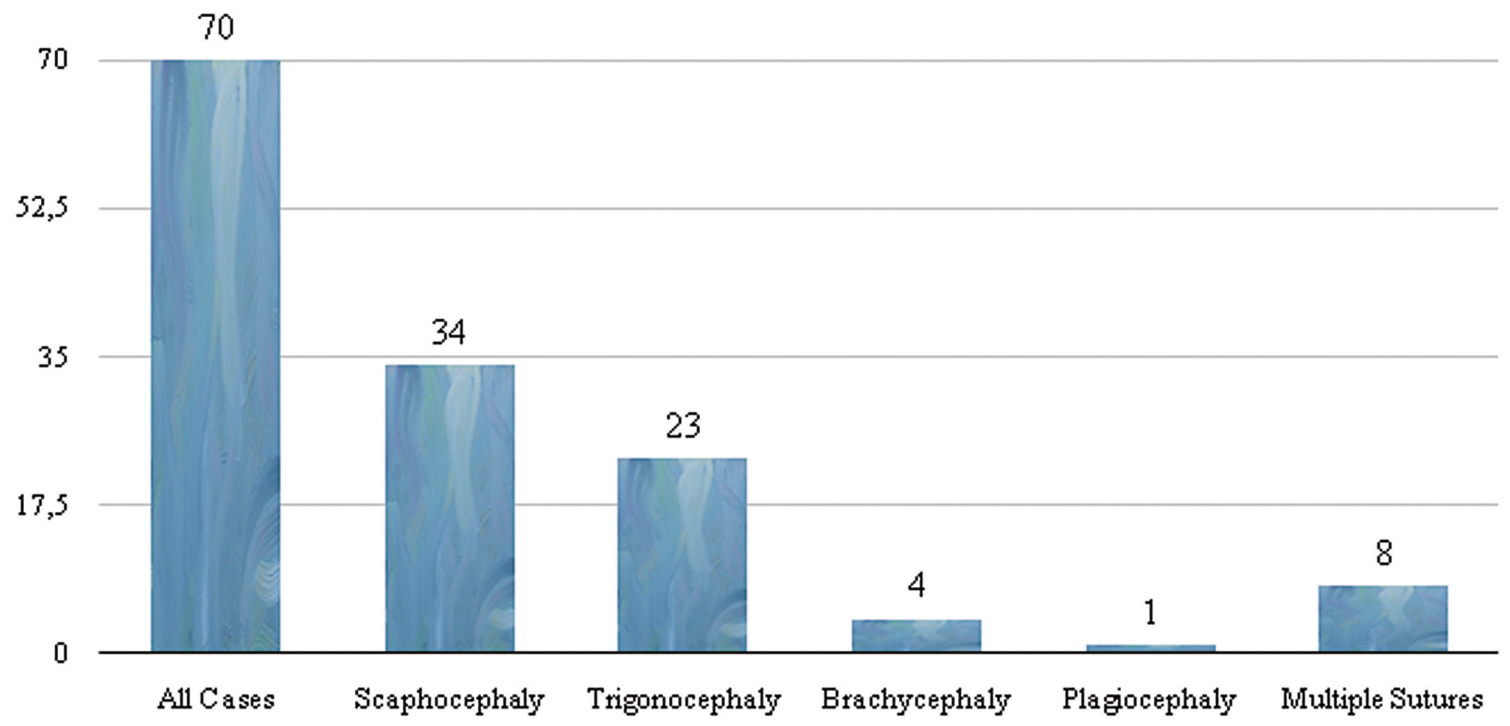

\section{Conclusion}

The results of recent studies report significantly low complication rates. The intraoperative outcomes presented in this series follow a similar trend with an overall complication rate of $12.7 \%$. With the ability to achieve the desired shape at the time of surgery and relatively low incidences of reoperation, as well as low morbidity and mortality rates, open cranial vault reconstruction remains a valuable method in the management of patients with craniosynostosis.

\section{Funding}

No funding was received for this study. 


\section{Conflict of Interests}

The authors declare no conflict of interest.

\section{Role of Author and Co-authors}

Evangelos Kilipiris (concept - design of the paper and writing) Frantisek Horn (material collection and writing) Michal Petrik (material collection and writing) Michal Kabat (material collection and writing) Jan Trnka (material collection and writing) Peter Stanko (editing)

\section{Ethical Approval}

Approval was obtained from the Medical Ethics Committee of the Comenius University in Bratislava.

\section{Patient Consent}

Written patient consent was obtained from parents to publish the clinical photographs.

\section{Acknowledgements}

None.

\section{References}

1. Zakhary G, Montes D, Woerner J, Notarianni C, Ghali G. Surgical correction of craniosynostosis. A review of 100 cases. J Cranio-Maxillo-Fac Surg 2014;42:1684-91.

2. Slater B, Lenton K, Kwan M, Gupta D, Wan D, Longaker M. Cranial sutures: a brief review. J Plast Reconstr Surg 2008; 121:170-8.

3. Ghali G, Sinn D, Tantipasawasin S. Management of nonsyndromic craniosynostosis. Atlas Oral Maxillofac Surg Clin North Am 2002;10:1-41.

4. Jiminez D, Barone C. Multiple suture noisyndromic craniosynostosis: early and effective management using endoscopic techniques. J Neurosurg Pediatr 2010;5:223-31.

5. Eppley B, Morales L, Wood R, Penslar J, Goldstein J, Havlik $\mathrm{R}$, et al. Resorbable PLLA-PGA plate and screw fixation in pediatric craniofacial surgery: clinical experience in 1883 patients. J Plast Reconstr Surg 2004;114:850-6.

6. Panchal J, Amirsheybani H1, Gurwitch R, Cook V, Francel P, Neas B, et al. Neurodevelopment in children with single-suture craniosynostosis and plagiocephaly without synostosis. J Plast Reconstr Surg 2001;108:1492-8. 